\title{
Infrared near-field microscopy with the Vanderbilt free electron laser: overview and perspectives
}

\author{
D. Vobornik ${ }^{\mathrm{a}, *}$, G. Margaritondo ${ }^{\mathrm{a}}$, J.S. Sanghera ${ }^{\mathrm{b}}$, P. Thielen ${ }^{\mathrm{b}}$, \\ I.D. Aggarwal ${ }^{\mathrm{b}}$, B. Ivanov ${ }^{\mathrm{c}}$, J.K. Miller ${ }^{\mathrm{c}}$, R. Haglund ${ }^{\mathrm{c}}$, N.H. Tolk ${ }^{\mathrm{c}}$, \\ A. Congiu-Castellano ${ }^{\text {d }}$, M.A. Rizzo ${ }^{\text {e }}$, D.W. Piston ${ }^{\text {e }}$, F. Somma ${ }^{f}$, \\ G. Baldacchini ${ }^{\mathrm{g}}$, F. Bonfigli ${ }^{\mathrm{g}}$, T. Marolo ${ }^{\mathrm{g}}$, F. Flora ${ }^{\mathrm{g}}$, R.M. Montereali ${ }^{\mathrm{g}}$,

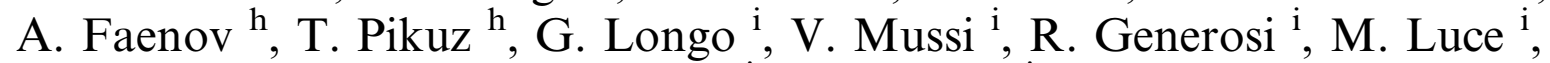 \\ P. Perfetti ${ }^{\mathrm{i}}$, A. Cricenti ${ }^{\mathrm{i}}$ \\ ${ }^{a}$ Faculté des Sciences de base, Ecole Polytecnique Fédérale de Lausanne (EPFL), CH-1015 Lausanne, Switzerland \\ ${ }^{\mathrm{b}}$ Optical Sciences Division, US Naval Research Laboratory, 4555 Overlook Ave SE, Washington, DC 20375, USA \\ ${ }^{\mathrm{c}}$ Department of Physics and Astronomy, Vanderbilt University, Nashville, TN 31235, USA \\ d Dipartimento di Fisica, Università di Roma "La Sapienza", 00185 Roma, Italy \\ ${ }^{\mathrm{e}}$ Department of Molecular Physiology and Biophysics, Vanderbilt University, Nashville, TN 37232, USA \\ ${ }^{\mathrm{f}}$ Dipartimento di Fisica, University Roma Tre, V. della Vasca Navale 84, 00146 Roma, Italy \\ ${ }^{\mathrm{g}}$ ENEA, UTSTecnologie Fisiche Avanzate, C.R. Frascati, Via E. Fermi 45, 00044 Frascati, Italy \\ ${ }^{\mathrm{h}}$ MISDC of VNIIFTRI Mendeleevo, Moscow region 141570, Russia \\ i Istituto di Stuttura della Materia, via Fosso del Cavaliere 100, 00133 Roma, Italy
}

Available online 20 March 2004

\begin{abstract}
Scanning near-field optical microscopy (SNOM) makes it routinely possible to overcome the fundamental diffraction limit of standard (far-field) microscopy. Recently, aperture-based infrared SNOM performed in the spectroscopic mode, using the Vanderbilt University free electron laser, started delivering spatially-resolved information on the distribution of chemical species and on other laterally-fluctuating properties. The practical examples presented here show the great potential of this new technique both in materials science and in life sciences.
\end{abstract}

(C) 2004 Elsevier B.V. All rights reserved.

\section{Introduction}

The main motivation for the development of scanning near-field optical microscopy (SNOM) is

\footnotetext{
${ }^{*}$ Corresponding author.

E-mail address: dusan.vobornik@epfl.ch (D. Vobornik).
}

beating the lateral resolution diffraction limit of conventional far-field microscopy [1-13]. This is of course particularly important for long wavelengths in the infrared (IR) range. Extensive tests did demonstrate IR lateral resolution well beyond the diffraction limit. The most attractive possibility is to perform IR SNOM experiments in a "spectroscopic" mode, by selecting the wavelength to excite 
specific vibration modes and to identify the spatial distribution of the corresponding chemical species [8,14-18]. Our present tests demonstrate, by coupling SNOM with the infrared radiation coming from the Vanderbilt free electron laser, that this is possible not only for materials-science systems but also for bio-systems.

Our work is based on the aperture-based SNOM approach. We note that a different "apertureless" SNOM approach was also developed (see Refs. [10-13,19]) that images different samples with nanometric resolution. Apertureless SNOM is very interesting for its potential applications to IR imaging in biology. However, contrary to aperture-based SNOM, the apertureless approach is not suitable for samples in a liquid environment, as frequently required in biology.

The results on bio-systems are particularly important. With spectroscopic SNOM, individual cells and specific features inside a cell become visible and can be investigated on a micron and submicron scale. This would be absolutely impossible within the diffraction limit, since the IR wavelengths are much larger than the imaged features.

Discovered by Abbé in the 19th century, the diffraction limit is linked to the very foundations of physics. According to the uncertainty principle, a photon cannot be localized in the transverse $(x)$ direction with accuracy better than $2 \pi /\left[(2 \pi / \lambda) 2-k_{y}\right]^{1 / 2}$, where $k_{y}$ is the longitudinal component of the $k$-vector. If $k_{y}$ is real, then the transverse resolution limit cannot exceed a limit of the order of $\lambda$. More accurately, if the Rayleigh criterion is adopted then the quantitative resolution limit is $\lambda / 2$.

This logic, however, is no longer valid if $k_{y}$ is imaginary, as in an evanescent or absorbed photon field. In a SNOM experiment, an imaginary longitudinal $k$-component is obtained by placing the tapered end (aperture $<\lambda$ ) of an optic fiber close (distance $<\lambda$ ) to the object. Many tests in the past several years confirmed that the diffraction limit can indeed be defeated in this way [1-10].

IR spectroscopic SNOM is one of the most powerful versions of near-field microscopy $[8,14$ 18]. Images are taken using transmitted or reflected IR light with a wavelength close to one of the vibrational modes of the specimen. By com- parison with images taken at other wavelengths, one can thus observe the lateral distribution of the chemical species corresponding to the vibrational mode. Unfortunately, the impact of spectroscopic SNOM was limited in the past by instrumentation problems.

Recent instrumentation advances - and in particular the use of a tunable free electron laser (FEL) as the IR source-removed most of these difficulties [10,14-21]. We present here successful IR spectroscopic SNOM experiments on different systems in materials science and the first tests in the life sciences.

\section{Experimental details}

Several versions of a specially designed multitechnique SNOM module [22] (located at the ISMCNR in Frascati, at the EPFL in Lausanne and at the Vanderbilt University Keck Foundation Free Electron Laser Center in Nashville) were used for our tests. The instrument is quite versatile, being able to take shear-force (topographic) images as well as different types of spectroscopic SNOM images. Note that the direct comparison of corresponding topographic images and SNOM images is essential to prove that no artifacts exist in the SNOM images.

IR photons from the unfocussed FEL beam or from other IR sources were directed onto the sample surface. The reflected photons were detected through a narrow-point optical fiber tip mounted on the SNOM module that also yielded the shear-force images [22].

The shear-force signal was independently used to keep the tip-surface distance constant while taking SNOM images. The shear force was detected by laterally oscillating the optical fiber while focusing on it a laser beam $(670 \mathrm{~nm})$. The resulting shadow was revealed by a two sector position sensitive detector placed on the opposite side. The alternating signal was revealed by an AC/DC converter and sent into the electronic feedback loop to keep a constant shear-force signal between fiber and sample while scanning the sample. Topographical and optical images were taken simultaneously, with the IR source illuminating 
the specimen over a broad area $(\sim 1 \mathrm{~mm}$ spot diameter) and the SNOM probe collecting the reflected light.

The fabrication of extremely high quality infrared fiber tips was a crucial technical step [23] in the practical realization of our experiments. Tapered IR SNOM probes were obtained from single-mode, 1-m long arsenic selenide fibers with $50 \mu \mathrm{m}$ clad diameter and $100 \mu \mathrm{m}$ core diameter. An adapter was fitted on one end of the fibers using phenyl salicylate as a solid support. Then, using water as a lubricant the adapter end of the fiber was polished to a smooth clean surface using rotating aluminum oxide discs with the final polish done at $0.3 \mu \mathrm{m}$. This end of the fiber was interfaced to the detector.

The other end of the fiber was chemically etched using a protective layer etching system. The etching (piranha) solution was $7: 3\left(\mathrm{H}_{2} \mathrm{O}_{2}: \mathrm{H}_{2} \mathrm{SO}_{4}\right)$. The etching typically took $30-45 \mathrm{~min}$ depending on the fiber diameter, etching concentration and temperature. An optical microscope was used to monitor the etching process. The etched tips were coated with gold using a Varian thermal deposition system at a pressure of $10^{-6}$ Torr-with the tips at $25-30^{\circ}$ above the evaporation point source to create an aperture. The tips were rotated to achieve a uniform coating with a thickness of 100-125 nm.

A key technical problem in IR spectroscopic SNOM is the IR source that must be tunable over a broad band and intense (to offset the limited fiber transmission). The best sources are FELs [15-17] and optical parametric amplifiers [24]. The Vanderbilt FEL is continuously tunable over a broad wavelength range $(2.1-9.8 \mu \mathrm{m})$, very intense and very reliable - and therefore ideal for IR spectroscopic SNOM.

\section{Materials science results}

Our first tests of IR spectroscopic SNOM were performed on artificial polycrystalline diamond films grown by plasma-assisted chemical vapor deposition (CVD) [25]. In principle, these films should be hydrogen-free after annealing to remove residual hydrogen and water. However, FTIR showed the presence of hydrogen (see Fig. 1, top).
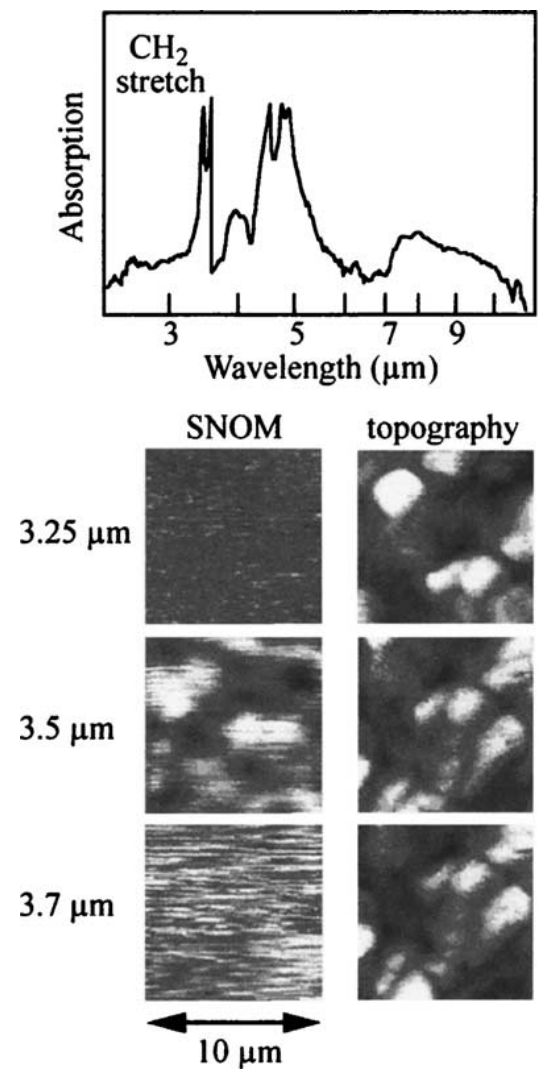

Fig. 1. Results (see Ref. [25]) on polycrystalline diamond films grown by plasma-assisted chemical vapor deposition (CVD). Top: absorption spectrum. Bottom: spectroscopic (reflectivity) SNOM images and shear-force topographic images taken with three different wavelengths. The $3.5 \mu \mathrm{m}$ wavelength corresponds to a $\mathrm{C}-\mathrm{H}$ vibrational stretch mode absorption band and reveals the presence of hydrogen.

We tested this point by tuning the FEL to a $\lambda=3.5 \mu \mathrm{m}$, corresponding to a $\mathrm{C}-\mathrm{H}$ vibrational stretch mode absorption band.

Fig. 1 (bottom, left-hand side) shows three $10 \times 10 \mu \mathrm{m}^{2}$ SNOM reflectivity images obtained at $\lambda=3.5 \mu \mathrm{m}$ and with two off-absorption wavelengths. On these images, darker areas correspond to lower light intensity collected by the SNOM fiber tip. Contrary to the on-absorption image, the two off-absorption images are featureless. The comparison with the corresponding shear-force (topological) image, on the bottom right-hand side of Fig. 1 can be used to assess the possible presence of artifacts. The dark regions of the on-absorption SNOM image indicate the presence of residual hydrogen mainly around grain boundaries. 
For the topographic images, line scans yield resolution estimates between 50 and $80 \mathrm{~nm}$, demonstrating the high quality of the fiber tips. Similar line scans for the spectroscopic SNOM images demonstrate a lateral resolution of $200 \mathrm{~nm}$, well beyond the diffraction limit.

This was the first successful test of IR spectroscopic SNOM and confirmed the FTIR results showing that hydrogen was in fact present in this nominally hydrogen-free system. The SNOM test provided information on the hydrogen distribution and opened the door to spectroscopic SNOM tests in other systems.

Fig. 2 shows results for a boron nitride film (with oxide components) on silicon. The objective in this case was to prove that spectroscopic SNOM could provide fine chemical and structural information on a microscopic scale. Specifically, the experiment targeted different vibrational modes corresponding to different crystallographic structures.

The FTIR spectrum of Fig. 2a reveals indeed several vibrational modes. Of these, the features at 7.4, 7.9 and $9.4 \mu \mathrm{m}$ are directly associated to the hexagonal, wurzite and cubic structures of BN. The spectroscopic (reflectivity) SNOM images of Fig. 2b-d clearly reveal differences in the lateral distribution of such phases. By comparison with the topographic image of Fig. 2e, we can see that, for example, the strongest contribution for the wurzite structure, Fig. 2c, arise from one side of the central big grain.

Fig. 2f shows an intensity profile along the line marked in Fig. 2b. The intensity changes can be due to topographic or spectroscopic causes. However, changes occur in areas where they cannot be justified by topography (compare Fig. $2 f$ and g). An extensive analysis of such changes in different line scans leads to a conservative estimate of a lateral resolution of at least $\lambda / 20$.

Fig. 3 shows the results of a third series of materials-science tests, dedicated to lithium fluoride films on silicon substrates. The objective in this case was not the lateral distribution of different chemical species but the lateral changes in the refractive index induced by X-ray bombardment [26].

$\mathrm{LiF}$ films are quite interesting as potential components of miniaturized optical devices. The
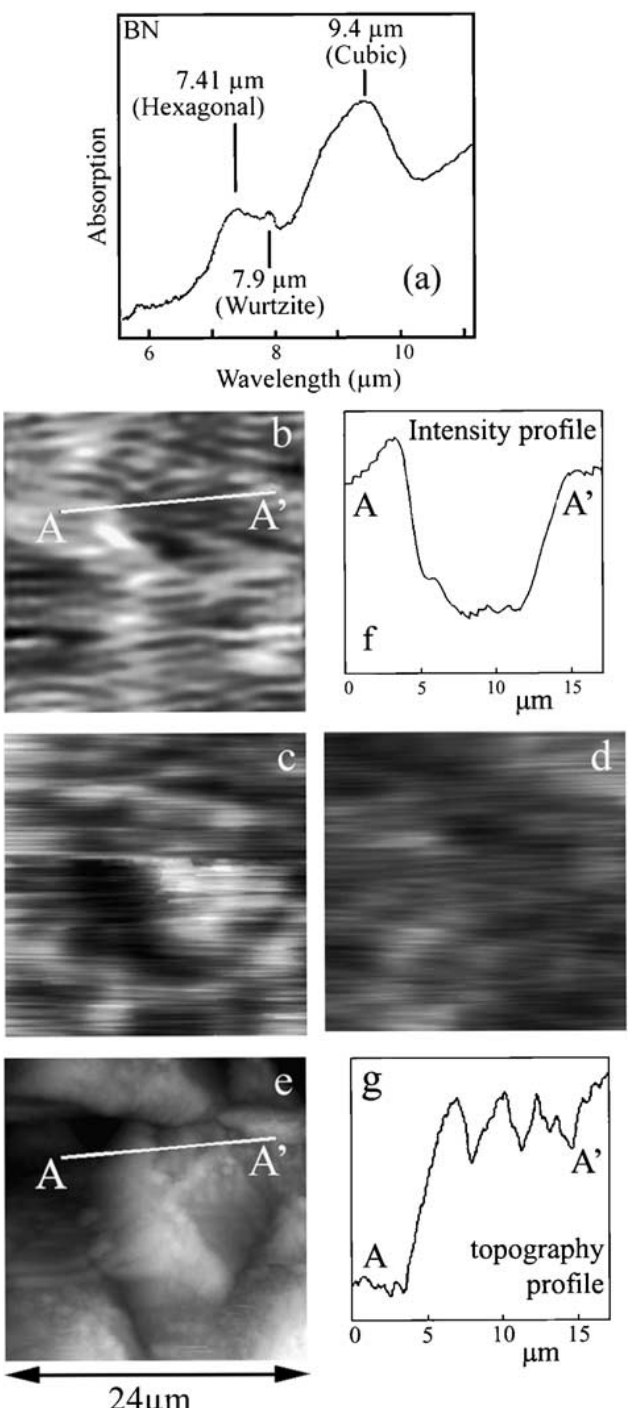

Fig. 2. Results on a BN film. (a) FTIR spectrum with the vibrational modes corresponding to three crystallographic phases. (b-d) Spectroscopic (reflectivity) SNOM images at the wavelengths 9.4, 7.9 and $7.41 \mu \mathrm{m}$. (e) Topographic image. (f,g) Intensity profiles for the marked line in Fig. $2 \mathrm{~b}$ and e.

creation of defects - called "color centers" [27] because of their influence on the color of the crystal - is a critical step in the corresponding fabrication procedure [28-31]. Such defects can be created by different processes; in the present case, we explored the results of an innovative irradiation technique [31] based on extreme ultraviolet (EUV) radiation and soft $\mathrm{X}$-rays emitted by a laser 
a

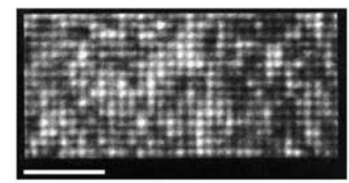

$100 \mu \mathrm{m}$
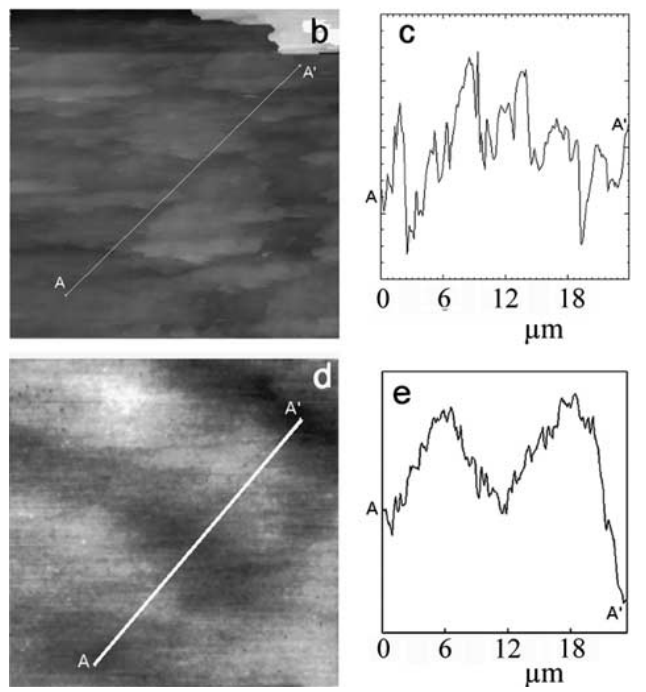

Fig. 3. Results on a LiF film on silicon with patterned coloring by X-ray irradiation (see Ref. [21]). (a) fluorescence optical micrograph of the colored pattern (the white spots correspond to irradiated areas). (b) $20 \times 20 \mu \mathrm{m}^{2}$ shear-force image. (c) Intensity profile along the marked line of Fig. 3b. (d) Spectroscopic (reflectivity) SNOM image taken with a wavelength of $6.1 \mu \mathrm{m}$. (e) Intensity profile along the marked line of Fig. $3 \mathrm{~d}$.

plasma-point source. This approach can produce patterned coloration on large areas.

High-quality LiF films (thickness $\approx 800 \mathrm{~nm}$ ) were grown by thermal evaporation on untreated silicon substrate 13 at $250{ }^{\circ} \mathrm{C}$. The films total thickness, measured by a stylus profilometer after the growth, was about $800 \mathrm{~nm}$. EUV and soft Xray radiation was produced by focusing an excimer laser beam (wavelength $=308 \mathrm{~nm}$ ) on a tapeshaped solid target in vacuum. The $\mathrm{LiF}$ was also in vacuum $\sim 12 \mathrm{~cm}$ from the plasma source; the irradiated area was several square centimeters. A colored pattern was obtained by placing on the LiF films a copper mesh (wire diameter $10 \mu \mathrm{m}$, period $12.7 \mu \mathrm{m}$ ).

Fig. 3a shows a fluorescence optical micrograph of the colored pattern; the white spots correspond to irradiated areas. Fig. $3 b$ is a $20 \times 20 \mu \mathrm{m}^{2}$ shearforce image showing LiF surface topography (see the corresponding profile in Fig. 3c). Fig. 3d shows the corresponding spectroscopic (reflectivity) SNOM image taken with a wavelength of $6.1 \mu \mathrm{m}$. At this wavelength we can clearly observe contrast in the image that is not correlated to the topographic SNOM features, and shows a periodicity corresponding to the pattern shown in Fig. 3a. Similar results were obtained with images taken at a wavelength of $9.2 \mu \mathrm{m}$. Thus, the search for irradiation-induced lateral fluctuations in the refractive index was successful. Since there is no special absorption in this spectral region we ascribe the change in reflectivity to be due to a variation of the refractive index of the exposed $\mathrm{LiF}$ material.

Fig. 3e shows an intensity profile along the marked line of Fig. 3d. From such profiles, we estimated a lateral resolution of $200-300 \mathrm{~nm}$ (50 $\mathrm{nm}$ in the topographic images), again much better than the diffraction limit corresponding to the 6.1 and $9.2 \mu \mathrm{m}$ wavelengths.

\section{Results on biological specimens}

Tests on cell systems were the biggest novelty of our study for the already mentioned reasons that prevent standard IR techniques from adequately contributing to the life sciences. So far, these tests concerned unmarked specimens [32] as well as systems treated with fluorescent markers (intestinal and neuron cells). We will present here successful tests on unmarked specimens.

The first experiments [32] were performed on thin films of a biofilm growth medium. A thick silicon substrate was submerged in a postgate growth medium solution at room temperature, then dipped in deionized water and allowed to dry for one day. The film-covered areas of the substrate were analysed first with an optical microscope and then with the SNOM system.

The growth medium has several constituents [33] - primarily sulfur and nitrogen compoundswhose vibrational stretch mode wavelength falls near $7 \mu \mathrm{m}$ [34]. IR Spectroscopic (reflectivity) SNOM images were taken at wavelengths of 6.95 and $6.6 \mu \mathrm{m}$, inside and outside the absorption region. 

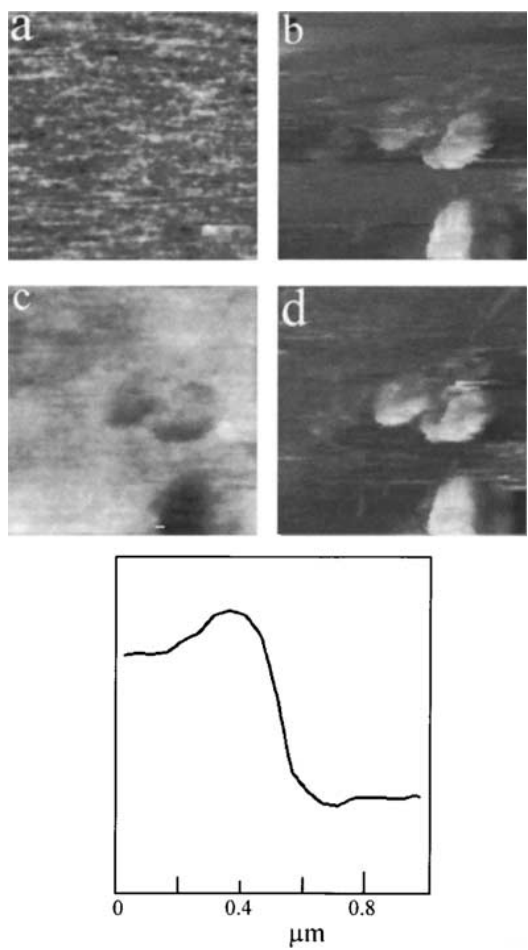

Fig. 4. Results on a thin films of a biofilm growth medium (see Ref. [32]). (a) $20 \times 20 \mu \mathrm{m}^{2} \mathrm{SNOM}$ reflection images obtained with $\lambda=6.6 \mu \mathrm{m}$, and (b) the corresponding topographic image. (c, d) Equivalent images for $\lambda=6.95 \mu \mathrm{m}$ corresponding to the vibrational stretch mode absorption bands of sulfur and nitrogen compounds. Darker areas correspond to stronger absorption. (e) Intensity profile taken along the marked line of Fig. 1c.

Fig. 4 shows $20 \times 20 \mu \mathrm{m}^{2}$ spectroscopic SNOM images together with the corresponding shearforce opography images. The contrast between the featureless off-absorption image and the rich microstructure in the on-absorption image is quite striking. Line scans like that of Fig. 4e reveal a resolution of $100 \mathrm{~nm}$ for the spectroscopic images (50-80 $\mathrm{nm}$ for the topographic images).

A second series of experiments (Fig. 5) was conducted on tissue culture cells from a rat pancreatic $\beta$ cell line (INS-1). Such cells were seeded on glass cover slides; after $24 \mathrm{~h}$ the medium was removed and the cells were fixed in paraformaldehyde and washed twice with PBS and twice with distilled water. The spectroscopic-SNOM images at $6.95 \mu \mathrm{m}$ (sulfide stretch band) and $6.1 \mu \mathrm{m}$
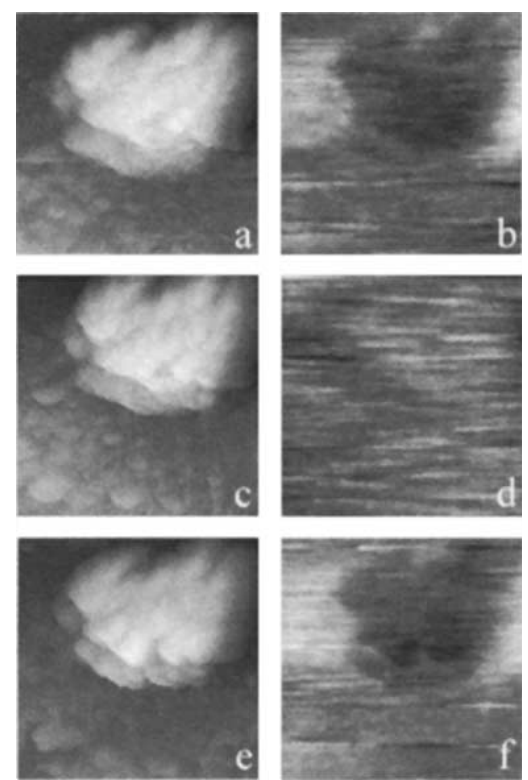

Fig. 5. $20 \times 20 \mu \mathrm{m}^{2} \mathrm{SNOM}$ reflection images of tissue culture cells from a rat pancreatic $\beta$ cell line (INS-1) in liquid (see Ref. [32]). $(a, b)$ topographic image and spectroscopic (reflectivity) image at a wavelength of $6.1 \mu \mathrm{m}$; (c,d) similar images for 6.45 $\mu \mathrm{m}$; (e,f) similar images for $6.95 \mu \mathrm{m}$.

(amide $\mathrm{I}, \mathrm{C}=\mathrm{O}$ stretch band) show absorption within the cell, whereas the $6.45 \mu \mathrm{m}$ (amide II band) image shows much less contrast.

The near featureless images taken at $6.45 \mu \mathrm{m}$ is quite surprising and the cause of the somewhat puzzling difference with respect to the Amide I band is not yet clear. We tested this experimental finding by taking many images with consistent results in all parts of the sample. Tentatively, the fact that absorption from the Amide II band is spread out throughout the sample could be due to uniform absorption from material on the coverslip, i.e., cells and precipitated crystals.

Fig. 6 directly emphasizes the impact of the IR near-field approach in the case of cell specimens. The top part is a replica of the Fig. $5 f$ image. The bottom part shows the same image after blurring corresponding to the diffraction limit at the wavelength of $6.1 \mu \mathrm{m}$. The message is quite clearand stresses the advantages offered by near-field techniques when using IR wavelengths in the life sciences. 

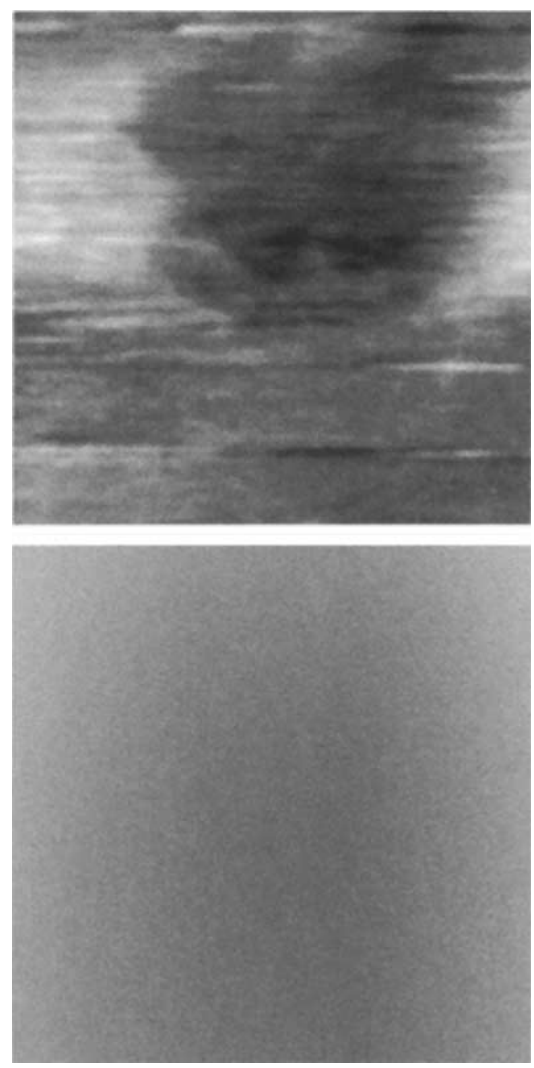

Fig. 6. A direct argument showing the importance of beating the diffraction limit with IR near-field techniques in the life sciences. Top: replica of the Fig. $5 f$ image; bottom: the same image after blurring corresponding to the diffraction limit at the wavelength of $6.1 \mu \mathrm{m}$. Within the diffraction limit, all information is lost at the cellular level

\section{Future perspectives}

The results presented here are just some of the first steps in the use of spectroscopic SNOM. The rapid improvements in the instrumentation will certainly enhance the capabilities of these techniques in the forthcoming years.

These improvements will specifically concern the light sources. The FEL technology is continuously improving and delivers better and better performances. Furthermore, infrared beam lines at conventional synchrotron sources could also play an important role.

Another important element in the evolution of this field will be the development of specific IR technique for the life sciences. So far, the technical limitation related to the diffraction limit made the efforts in this direction somewhat futile. This was changed by the near-field strategy, but the message did not yet reach the potential users in the life sciences. A direct interdisciplinary collaboration is thus necessary to make full use of the remarkable new capabilities illustrated by the examples presented here.

\section{Acknowledgements}

We would like to thank the entire staff of the W.M. Keck Foundation FEL Center at Vanderbilt University for their able assistance and Vinh Nguyen (NRL) and Paul Pureza (NRL) for help in making the optical fibers. This work is supported by the Italian National Research Council (CNR), Ecole Polytechnique Federale de Lausanne, the Fonds National Suisse de la Recherche Scientifique, the National Institutes of Health, the US Office of Naval Research and the US Air Force Office of Scientific Research.

\section{References}

[1] E.A. Ash, G. Nicholls, Nature 237 (1972) 510.

[2] D.W. Pohl, W. Denk, M. Lanz, Appl. Phys. Lett. 44 (1984) 651.

[3] E. Betzig, J.K. Trautman, Science 257 (1992) 189.

[4] E. Betzig, P.L. Finn, J.S. Wiener, Appl. Phys. Lett. 60 (1994) 2484

[5] H. Heinzelmann, D.W. Pohl, Appl. Phys. A 59 (1994) 89.

[6] D.W. Pohl, D. Courjon (Eds.), Near Field Optics, NATO ASI Series, vol. 262, Kluwer Academic Press, 1992.

[7] J. Almeida, T. dell'Orto, C. Coluzza, G. Margaritondo, O. Bergossi, M. Spajer, D. Courjon, Appl. Phys. Lett. 69 (1996) 2361

[8] A. Cricenti, R. Generosi, P. Perfetti, J.M. Gilligan, N.H. Tolk, C. Coluzza, G. Margaritondo, Appl. Phys. Lett. 73 (1998) 151.

[9] A. Cricenti, G. Longo, M. Luce, R. Generosi, P. Perfetti, D. Vobornik, G. Margaritondo, P. Thielen, J.S. Sanghera, I.D. Aggarwal, J.K. Miller, N.H. Tolk, D.W. Piston, F. Cataruzza, A. Flamini, T. Prosperi, A. Mezzi, Surf. Sci. 544 (2003) 51.

[10] B. Knoll, F. Keilmann, Nature 399 (1999) 134.

[11] S. Gresillon, S. Ducourtieux, A. Lahrech, L. Aigouy, J.C. Rivoal, A.C. Boccara, Appl. Surf. Sci. 164 (2000) 118.

[12] R. Bachelot, P. Gleyzes, A.C. Boccara, Appl. Opt. 36 (1997) 2160. 
[13] L. Stebounova, B.B. Akhrenmitchev, G.C. Walker, Rev. Sci. Instrum. 74 (2003) 3670.

[14] A. Piednoir, C. Licoppe, F. Creuzet, Opt. Commun. 129 (1996) 414.

[15] D.V. Palanker, G.M.H. Knippels, T.I. Smith, H.A. Schwettman, Nucl. Instr. Meth. Phys. B 144 (1998) 240.

[16] M.K. Hong, A.G. Jeung, N.V. Dokholyan, T.I. Smith, H.A. Schwettman, P. Huie, S. Erramili, Nucl. Instr. Meth. B 144 (1998) 246.

[17] A. Cricenti, R. Generosi, C. Barchesi, M. Luce, M. Rinaldi, C. Coluzza, P. Perfetti, G. Margaritondo, D.T. Schaafsma, I.D. Aggarwal, J.M. Gilligan, N.H. Tolk, Phys. Status Solidi 170 (1998) 241.

[18] B. Dragnea, S.R. Leone, Int. Rev. Phys. Chem 20 (2001) 59.

[19] F. Zenhausern, Y. Martin, H.K. Wickramasinghe, Science 269 (1995) 1083.

[20] E. Tuncel, J.L. Staehli, C. Coluzza, G. Margaritondo, J.T. McKinley, R.G. Albridge, A.V. Barnes, A. Ueda, X. Yang, N.H. Tolk, Phys. Rev. Lett. 70 (1993) 4146.

[21] C. Coluzza, E. Tuncel, J.L. Staehli, P.A. Baudat, G. Margaritondo, J.T. McKinley, R.G. Albridge, A.V. Barnes, A. Ueda, N.H. Tolk, D. Martin, F. MorierGenoud, C. Dupuy, A. Rudra, M. Ilegems, Phys. Rev. B 46 (1992) 12834.

[22] A. Cricenti, R. Generosi, C. Barchesi, M. Luce, M. Rinaldi, Rev. Sci. Instrum. 69 (1998) 3240.

[23] D. Talley, L.B. Shaw, J.S. Sanghera, I.D. Aggarwal, A. Cricenti, R. Generosi, M. Luce, G. Margaritondo, J.M. Gilligan, N.H. Tolk, Mater. Lett. 42 (2000) 339.
[24] C.A. Michaels, S.J. Stranick, L.J. Richter, R.R. Cavanagh, J. Appl. Phys. 88 (2000) 4832.

[25] A. Cricenti, R. Generosi, M. Luce, P. Perfetti, G. Margaritondo, D. Talley, J.S. Sanghera, I.D. Aggarwal, J.M. Gilligan, N.H. Tolk, J. Microsc. (Oxford) 202 (2001) 446, and the references therein.

[26] A. Cricenti, G. Longo, V. Mussi, R. Generosi, M. Luce, P. Perfetti, D. Vobornik, G. Margaritondo, P. Thielen, J.S. Sanghera, I.D. Aggarwal, N.H. Tolk, G. Baldacchini, F. Bonfigli, F. Flora, T. Marolo, R.M. Montereali, A. Faenov, T. Pikuz, unpublished results.

[27] U.M. Grassano, G. Margaritondo, R. Rosei, Phys. Rev. B 2 (1970) 3319.

[28] R.M. Montereali, M. Piccinini, E. Burattini, App. Phys. Lett. 78 (2001) 4082.

[29] K. Schwartz, C. Trautmann, T. Steckenreiter, O. Greiss, M. Kramer, Phys. Rev. B 58 (1998) 17.

[30] C. Vigreux, P. Loiseau, L. Binet, D. Gourier, Phys. Rev. B 61 (2000) 13.

[31] G. Baldacchini, F. Bonfigli, F. Flora, R.M. Monatereali, D. Murra, E. Nichelatti, A. Faenov, T. Pikuz, Appl. Phys. Lett. 80 (2002) 4810.

[32] A. Cricenti, R. Generosi, M. Luce, P. Perfetti, G. Margaritondo, D.B. Talley, J.S. Sanghera, I.D. Aggarwal, N.H. Tolk, Phys. Chem. Chem. Phys. 4 (2002) 2738.

[33] D. de Beer, P. Stoodley, F. Roe, Z. Lewandowski, Biotechnology and Bioengineering 43 (1994) 1131.

[34] J.H. van der Maas, Basic Infrared Spectroscopy, second ed., Heyden, London, 1972, 88. 